\title{
Las estructuras construidas para la gestión del agua en los primeros poblados del Neolítico del Próximo Oriente: Aportación desde el estudio del asentamiento de tell Halula (Valle del Éufrates, Siria) Water management structures found in first Neolithic village at Near East: contribution from tell Halula (Euphrates Valley, Syria)
}

\author{
Miquel Molist ${ }^{1}$ \\ Anna Gómez ${ }^{1}$
}

\begin{abstract}
Resumen
Este artículo se centra en las dificultades para identificar e interpretar las estructuras relacionadas con la gestión del agua en asentamientos neolíticos (paredes y estructuras de adobe, suelos de yeso...). El yacimiento de Halula (Valle del Éufrates, Siria) es el caso de estudio utilizado para será analizado profundamente durante el periodo PPNB (hacia 7500 cal ANE). Particularmente las excavaciones desarrolladas en 2011 nos permitieron detectar áreas pobladas con evidencias de gestión de aguas residuales que han aportado un conjunto importante de muestras analizables. La interacción entre el agua, los espacios construidos y los espacios de circulación en áreas domésticas se vuelve esencial para comprender las estrategias adoptadas por estas comunidades y la respuesta de la arquitectura de tierra. Se expondrán estructuras complementarias como pozos, canalizaciones o depósitos para el almacenaje del agua se expondrán con el objetivo de validar la interpretación de la gestión del agua aplicando nuevas metodologías.
\end{abstract}

Palabras clave: Neolítico, Próximo Oriente, gestión del agua, Tell Halula.

\begin{abstract}
This paper focuses on difficulties to identify and interpret structures related to water management in Neolithic settlements (mudbricks, plasters floors...). Tell Halula (Euphrates Valley, Syria) as a case of study will be deeply analyzed during PPNB (c. 7500 cal ANE). Particularly the field work excavations developed on 2011 let us to detect populated areas rejection of wastewater and offered several samples to be studied. The interaction between water, built spaces and corridors in domestic areas become essential to understand water strategies from these communities and their mud-brick architecture. Complementarily, building structures such as wells, pipes or water deposits, will be presented with the aim to validate the water management interpretation using new methodologies.
\end{abstract}

Keywords: Neolithic, Near East, water management, Tell Halula.

\section{INTRODUCCIÓN}

La extensa investigación y desarrollo de la actividad arqueológica en los últimos decenios, en amplias áreas del Próximo Oriente, ha impulsado de manera significativa el conocimiento de los primeros poblados agrícolas. Aunque una buena parte de los resultados de los proyectos están en curso de análisis y publicación, muchos de los hallazgos están dinamizando y renovando la investigación del Neolítico en las diferentes áreas o campos temáticos y analíticos a través de los restos arqueológicos. En esta contribución nos proponemos realizar una aproximación a las estructuras construidas relacionadas con la gestión y uso del agua en las estruc- 
turas de hábitat de los poblados en la zona del Próximo Oriente ${ }^{2}$.

La revisión no quiere ser exhaustiva sino más bien focalizar en las novedades más importantes en la investigación de los sistemas de gestión de captación y evacuación de agua, primero, a nivel general y en una segunda parte, a través del análisis de los documentos recuperados en el proyecto desarrollado por el equipo en el yacimiento de Tell Halula (Valle del Éufrates, Siria).

Nos limitaremos a la caracterización de los elementos materiales constructivos con el objetivo de evaluar el grado de "domesticación" de los recursos hídricos en las áreas productivas y de las actividades domésticas realizadas en las construcciones y espacios destinados al hábitat. Su observación nos permitirá aproximarnos a la caracterización y análisis de las técnicas constructivas, observando tanto el grado de dificultad técnica, la especificidad o no de los materiales y elementos constructivos utilizados y su inserción en los ámbitos constructivos domésticos específicos. A la caracterización y análisis tecnológico se unirá una aproximación a la funcionalidad a pesar de las dificultades que entraña este último aspecto.

Dada la importancia del agua en las comunidades humanas el acceso de los recursos hídricos en relación a la aparición de las primeras sociedades agrícolas, su estudio y análisis ha sido un elemento clave en la historiografía de esta transformación social y económica. En primer lugar desde una aproximación medioambiental definiendo ámbitos ecológicos favorables a las prácticas agrícolas, siendo el índice de pluviosidad una de las variables clave para la producción controlada de plantas $\mathrm{y}$ frutos vegetales.

Pero la importancia al acceso y disponibilidad del agua para los primeros grupos de agricultores/ganaderos es mucho más amplia y la prueba más evidente es modelo de distribución de los asentamientos estando en general ubicados en las terrazas fluviales, en las áreas de desembocadura, o uadis o en las zonas de marismas. La disponibilidad de agua dulce en el asentamiento o en su proximidad es imprescindible para el desarrollo del poblado, es normal pues que se estudie la gestión del agua en los poblados de las primeras comunidades agro pastorales en una zona geográfica tan diversa, a nivel geográfico, geológico e hidrológico, como es el Próximo Oriente. Esta temática ha constituido un eje de nuestra investigación al cual se ha dedicado esfuerzo, tanto en el estudio de las evidencias arqueológicas como en el análisis de laboratorio.

\footnotetext{
2 Con ello queremos testimoniar nuestro más sincero reconocimiento a la Profesora Isabel Rubio, cuya dedicación al análisis y estudio del periodo Neolítico ha sido constante tanto a nivel de la Península Ibérica como en general. Su apoyo durante la ejecución del proyecto en Tell Halula fue constate, con su complicidad y la de los demás profesoras y profesores del Departamento de la UAM, en particular Concha Blasco y Javier Baena, donde fuimos invitados de manera regular a exponer los resultados científicos obtenidos de manera progresiva. Gracias Isabel por tu apoyo, trabajo y amistad.
}

En efecto, como es conocido el proyecto de investigación desarrollado en tell Halula (Valle del Éufrates, Siria) con una excavación estable de un asentamiento de los VIII-VI milenio y los estudios científicos derivados e interdisciplinares, permite tener una base documental excelente para la revisión de las relaciones y estructuras organizativas de un poblado de las primeras fases del Neolítico en relación a los recursos hídricos. Esta problemática ha sido tratada de manera directa en los diferentes estudios ya realizados sobre todo de tipo paleobotánico donde la incidencia del agua en el proceso agrícola ha sido reseñada con mayor detalle. Por una parte, en los estudios de R. Buxó y de G. Willcox, N. Rovira con los análisis carpológicos que establecieron que se trataba de una agricultura de secano, desarrollando el cultivo de cereales y leguminosas a lo largo de toda la secuencia neolítica (Willcox et al., 2009; Buxó y Rovira, 2013) Por otra parte se los trabajos de J.L. Araus, J. Voltes, P. Fierro, R. Buxó, sobre las necesidades hídricas para el cultivo de las plantas domésticas demostró las inferencias y aportaciones directas sobre las variaciones climáticas y la definición del medioambiente (Araus et al.. 1999; Fierro et al., 2011). También hay que destacar los estudios más recientes en arqueozoología, realizados pro M. Saña y C. Tornero, con la metodología analítica de los isotopos estables, analizando la alimentación de las especies animales domésticas explotadas infiriendo en el entorno medioambiental y los desplazamientos de los rebaños (Tornero y Saña, 2010). Finalmente y en esta misma dirección, aunque de manera más indirecta, el estudio de contenedores y soportes també ha permitido trabajar aspectos de volúmenes, porosidad y resistencia mecánica, teniendo en la producción cerámica para las etapas más recientes del yacimiento, su mayor área de estudio (Gómez-Bach, 2011; Cruells, 2013; Faura, 2016).

\section{Estudios y precedentes en el Próximo Oriente}

\subsection{La visión clásica}

La inferencia del factor hídrico ha estado presente en la literatura des de los trabajos iniciales de Braidwood (Braidwood y Braidwood, 1960). Autores como James Mellaart, Jacques Cauvin y sobretodo Olivier Aurenche, habían planteado este tema en las diferentes síntesis realizados entre los años 70 y 80 del siglo XX. Así por ejemplo el análisis de O. Aurenche $(1981,1982)$ sobre los dispositivos particulares en el espacio doméstico de las sociedades agrícolas tiene un capítulo dedicado a "amenagements pour l'eau" donde se incluyen las evidencias de pozos ("Puits"), de canales ("canaux") y de evacuación de agua ("l'evacuatión de l'eau"). Si bien las evidencias citadas son escasas y en general cuentan con una documentación poco descriptiva.

La orientación del análisis realizada por Jacques Cauvin es distinta al incidir, de forma más precisa, en las características medioambientales y la definición, más detallada, del área "nuclear" o zona donde se desarrolla 
la agricultura incipiente (Cauvin, 1981). De hecho este autor remarca que la ubicación de los primeros poblados agrícolas se documenta sobretodo en la zona de cultivo de secano en la proximidad de los puntos donde el agua es abundante. Esta evidencia le permite insistir a favor de la tesis de que el origen de la agricultura es una opción de tipo socio-cultural (Cauvin, 1994). Las obras de James Mellaart $(1965,1970)$ también recogen algunas de las evidencias de estructuras vinculadas con el uso de agua. De hecho recoge esencialmente las que fueron descubiertas en las excavaciones y proyectos dirigidos por él mismo en la zona de Anatolia. Así por ejemplo menciona en Hacilar la presencia de un pozo con las paredes revestidas de piedra y con un diámetro en la parte superior de $1,70 \mathrm{~m}$. Se trata de una estructura emplazada en el exterior de un patio en el nivel VI al nivel II del período 6 (Mellaart, 1970).

Agrupando las evidencias que en esta fase de la investigación se documentan, hay que citar por ejemplo la canalización para la evacuación del agua de Jericó (PPNB) y Bouqras (PPNB). En este último asentamiento H. Contenson descubrió una canalización en "terre de brique", en ligera pendiente hacia el sur-oeste, asociada a vestigios de suelos (Contenson y Van Liere, 1966: 184). Ligeramente más recientes son las evidencias documentadas en Byblos (c. 5500 cal ANE) donde en un suelo enlucido situado cerca de la entrada se documenta una ligera pendiente hacia la mitad de un muro (concretamente el sur) entrando para formar una salida de agua. (Dunand, 1973: 18). También en el yacimiento de Hassuna (nivel IV/ C. 5000 cal ANE) una estructura negativa a modo de surco se inicia en un hogar, con piedras reforzando su paso a través del muro del patio (Lloyd et al., 1945).

En resumen pocas evidencias muy a menudo mal documentadas tanto en las fases recientes del horizonte PPNB y ligeramente más abundantes en el horizonte del Neolítico cerámico: Hassuna, Chagar Bazar, Arpachiyah, bien recogidas en los trabajos del profesor O. Aurenche en su extensa literatura (a partir de Aurenche, 1981).

\subsection{Novedades recientes en la investigación en el Próximo Oriente}

El aumento de los trabajos de campo y el gran dinamismo en los estudios, en las últimas décadas, ha permitido aumentar de manera muy importante las evidencias arqueológicas de las instalaciones vinculadas a la gestión de los recursos hídricos, que en términos generales refuerzan las evidencias existentes y al mismo tiempo innovan en el conocimiento de sus características morfológicas, tecnológicas y variabilidad constructivas.

En el apartado de las estructuras destinadas a la captación de agua, destacan en primer lugar las evidencias de pozos. La localización de este tipo de estructuras en los asentamientos neolíticos de Chipre constituyen una gran novedad pues se trata de evidencias bien documentadas de estructuras subterráneas para la obtención de agua y documentadas en las fases arcaicas del Neolítico. Se trata de dos yacimientos chipriotas: Shillourocambous y Kissonerga-Mylouthkia con estructuras pertenecientes a las primeras ocupaciones de poblaciones neolíticas en la isla. Los pozos de Shillourocambous (st. 2, 66, 114), con una cronología de 8200-7900 Cal BC, están situados en el exterior de las casas y presentan un diámetro de 1 metro con unas profundidades variables entre 4,40-5,80 metros. Interpretados como un bien de uso comunitario (Guilaine, 2003; Guilaine et al., 2011). En el yacimiento de Khissonerga- Mylouthkia (Chipre), de cronología contemporánea, también se descubrieron varias estructuras similares (Structura 110 y 133) interpretadas como pozos para la captación de agua potable (Peltenburg, 2003).

En los periodos más recientes también citaremos las evidencias descubiertas recientemente en los asentamientos de la zona de la costa mediterránea. Es el caso de Israel, con el yacimiento de Atlit'Yam con una cronología de 7000 cal ANE (Galili et al., 1993) y sobretodo Sha'ar Hagolan con 3 pozos ubicados en el exterior de las construcciones domesticas en los niveles datados entre 64006200 cal ANE (Garfinkel et al., 2005).

En resumen, los últimos descubrimientos documentan estructuras de tipo pozo de una gran profundidad, con importantes trabajos de mantenimiento y reconstrucción que indican no solo un uso continuado sino también una inversión de trabajo significativo. Es muy interesante la documentación reciente de instalaciones para la captación de agua en superficie, de tipo embalse, de nuevo en los periodos recientes del PPNB. Se trata de estructuras con poca inversión de trabajo, pero con evidencias claras que permiten su interpretación para la finalidad de tener acceso a puntos de agua potable, ya sea para uso animal o humano. Su ubicación geográfica es también significativa pues están localizadas sobre todo en las áreas más áridas del Levante sur como en Jafr Basin (Jordania) o en Wadi Abu Tulayha (Jordania) siempre en cronologías de M/ LPPNB (Fujji, 2007, 2010).

El tipo de instalación que cuenta actualmente con un número mayor de evidencias son las estructuras construidas vinculadas con el uso o la evacuación del agua en el interior de las construcciones domésticas. En el presente artículo prestaremos una particular atención a este tipo de estructuras centrándonos en los documentos del yacimiento de Tell Halula (Valle del Éufrates, Siria) comparando con las evidencias similares en los yacimientos que por cronología y área geográfica son complementarios.

\section{Aportaciones del estudio de Tell Halula (Valle del Éufrates, Siria)}

Se expone a continuación los elementos estructurales en el asentamiento arqueológico de Tell Halula que permiten restituir la gestión del agua dentro del espacio domestico construido. Se trata de analizar los restos arqueológicos vinculados a la obtención pero sobre todo a la circulación y distribución del agua en las diferentes 


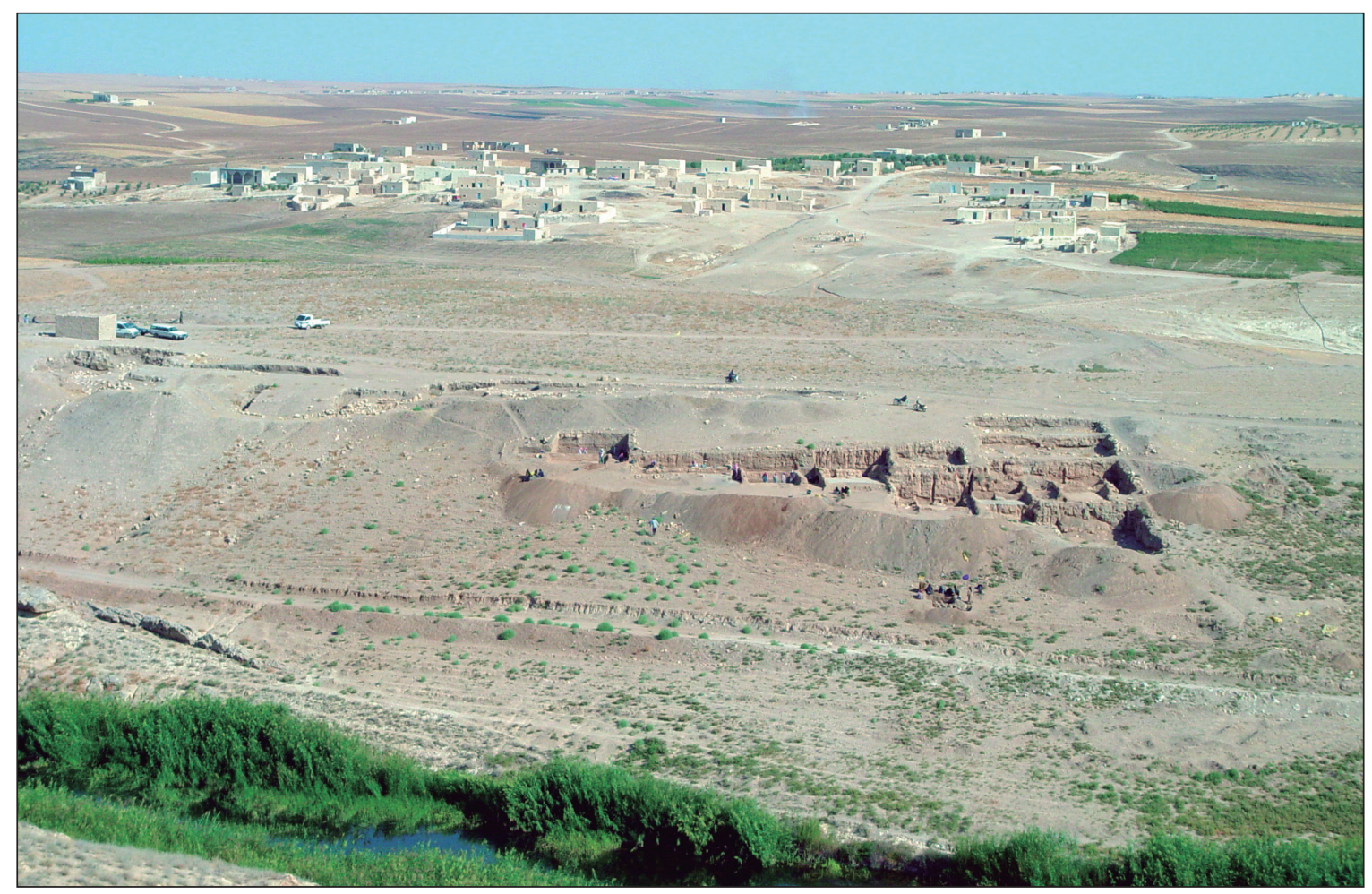

Figura 1. Vista general de la zona excavada en la parte sur de Tell Halula (Foto: SAPPO-UAB).

unidades domésticas o de las distintas estructuras de uso colectivo localizado en el asentamiento ${ }^{3}$.

Hasta el presente, no se tienen evidencias de estructuras construidas vinculas a la búsqueda y obtención del agua en el yacimiento. La proximidad del propio rio Éufrates pero sobretodo de los afluentes Wadi Narh el Fars y el Wadi Abu $\mathrm{Gal} \mathrm{Gal}^{4}$ que rodean el asentamiento, potencian la hipótesis de una recogida directa de la misma para el uso doméstico para el grupo humano. Probablemente las necesidades hídricas para la gestión de los rebaños fueran también por acceso directo en el curso de las actividades domésticas y de abastecimiento realizadas en el poblado (fig. 1).

\subsection{Sistemas de canalizaciones en espacios construidos}

Es en el ámbito del espacio doméstico donde se ha localizado un conjunto de estructuras que permiten infe-

Como es ampliamente conocido el proyecto de investigación de Tell Halula se ha llevado a cabo entre 1991-2011 por el equipo de investigación del SAPPO del Departamento de Prehistoria de la Universidad Autónoma de Barcelona, dirigido por el Dr. Miquel Molist. En el presente trabajo se analizan elementos arquitectónicos recuperados en las diferentes campañas de trabajo de campo y que fueron excavados por los diferentes miembros del equipo a lo largo de los 20 años de excavación. Reciban todos ellos y ellas nuestro más sincero agradecimiento.

En nuestro primer año de excavación, en septiembre de 1991, aun se pudo constatar la presencia de un pequeño curso de agua en el Wadi Abu Gal Gal, con la presencia de fauna estable de ambiente de ribera (cangrejos, ranas, ...). rir en la utilización del agua para labores domésticas del grupo humano. El análisis de los elementos específicos destinados a la circulación del agua y a la construcción, mantenimiento y reformas de estas instalaciones en el poblado se han documentado esencialmente entre las fases de ocupación 07 a 14 (c.7800-7200 cal ANE) del sector $2 / 4$ (sur del yacimiento), donde el conocimiento de la arquitectura y la disposición de las estructuras del poblado han sido excavadas con mayor amplitud y, por tanto, presentan un nivel de conocimiento superior (fig. 2).

En general, se trata de estructuras construidas o excavadas asociadas a la propia unidad de habitación. Se caracteriza principalmente por formar elementos construidos de manera regular y sistemática como parte de los dispositivos complejos para la evacuación del agua desde la habitación principal de las casas hacia el exterior de la construcción. En efecto, se ha documentado un sistema complejo que permite conocer las estructuras destinadas a la evacuación del agua o líquidos desde el interior de una casa hacia un lugar alejado de la casa.

Más concretamente, las evidencias se han documentado en las casas pertenecientes a las fases FO 9, 10 y 11 (c. 7600-7300 cal ANE). Agrupando toda la documentación se puede inferir en un sistema constructivo formado por los siguientes elementos:

En todos los casos el elemento básico es un agujero o apertura localizada en el muro lateral de la casa. Se trata de una perforación ubicada en uno de los ángulos de la habitación principal, muy cerca del suelo, éste está construido con una preparación compacta de cal, completado con un enlucido. En algún caso la perforación se 


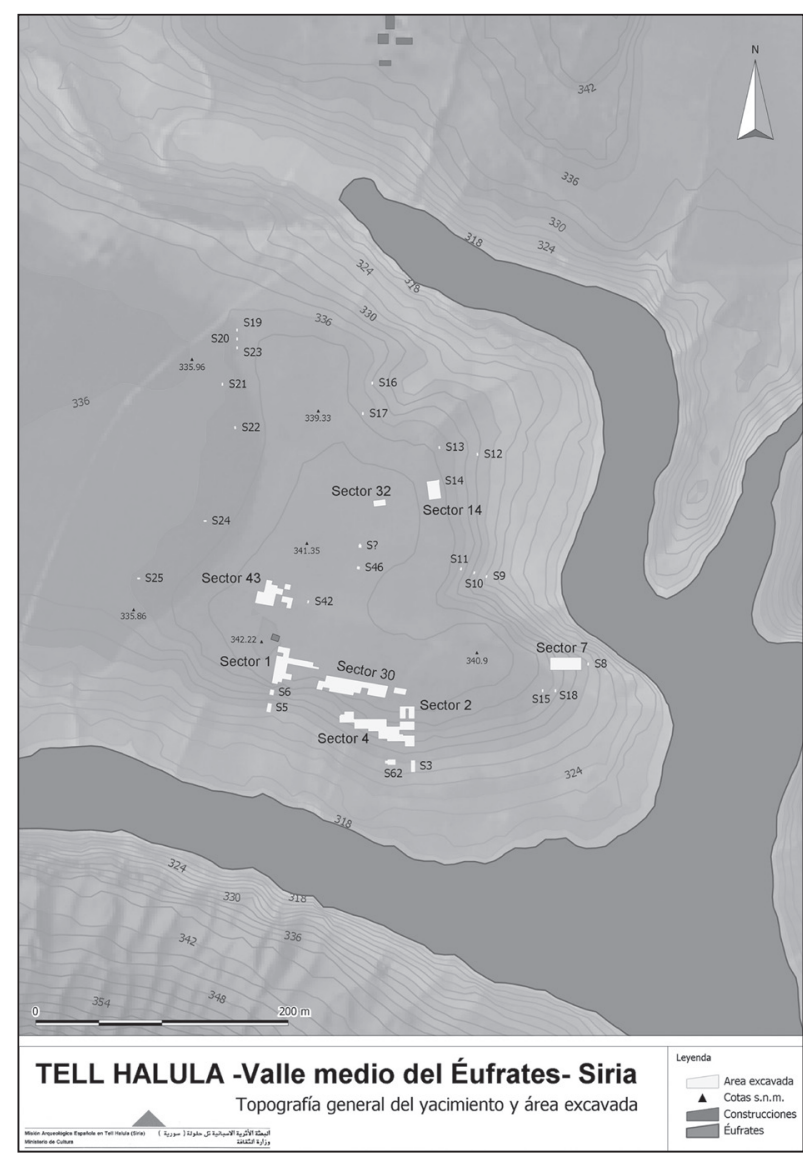

Figura 2. Topografia del Yacimiento con la indicación de las áreas de excavación (doc. SAPPO-UAB).

documenta en la parte media del muro exterior, pero la morfología y el sistema es idéntico. Este agujero tiene una sección circular o ligeramente ovalada y de un diámetro muy pequeño $(7-10 \mathrm{~cm}$.).

Un conducto de sección circular o ligeramente ovalada atraviesa el muro, llegando a la parte exterior del mismo ya en el exterior de la casa. No se ha documentado ningún tipo de arreglo reparación o reforma constructiva a este conducto. En el recorrido del interior del muro, en ningún caso, está enlucido con cal ni tampoco presenta protección de losas o gravilla en la base. Puede sorprender esta falta de elaboración pero habría que destacar que el diámetro es reducido y probablemente dificulta una mayor inversión en sus acabados. También nos podemos preguntar si estos elementos están previstos desde el inicio de la construcción de la casa, ya que parecen ser estructurales y relacionados con la habitación principal. Varios elementos como la regularidad de su presencia en las unidades de habitación y la homogeneidad de su morfología nos inclinan a pensar en una propuesta afirmativa y considerar que, en el modelo estándar de las construcciones, se prevé ya este elemento.

El pequeño canal, una vez atravesado el muro, llega al espacio exterior o callejón, desembocando en una estructura o fosa construida (a modo de estructura negativa) en los espacios exteriores a las unidades de habitación. Esta

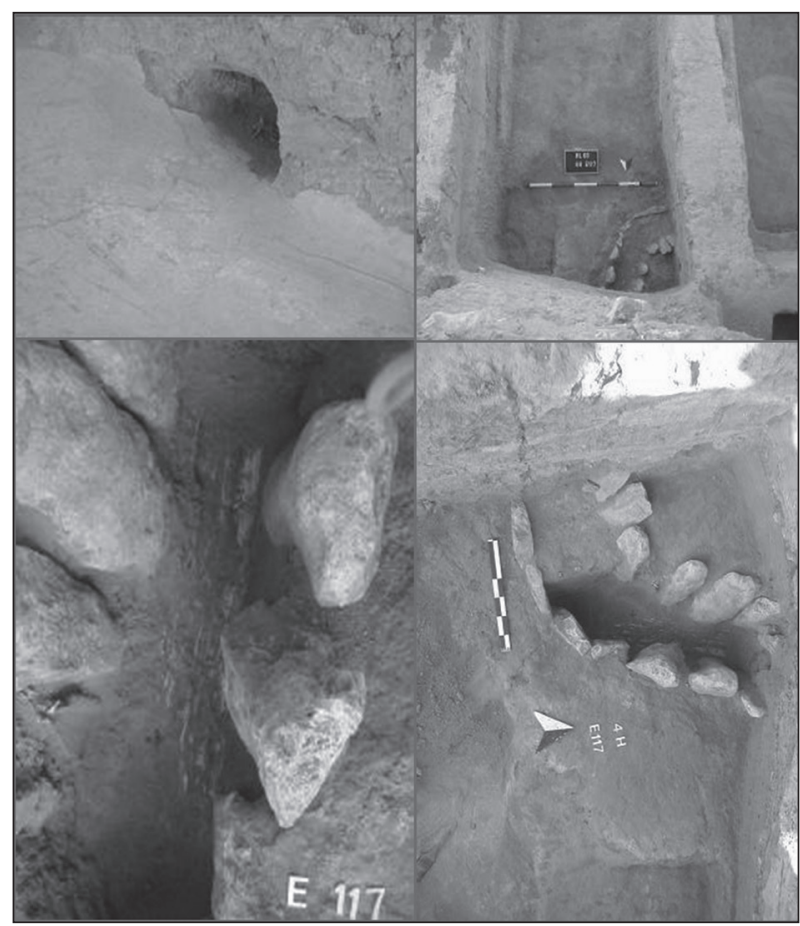

Figura 3. Tell Halula F.O. XII casa 4H, Canal de drenaje externo E 117. Cuatro imágenes de la instalación para la evacuación del agua del hábitat. (Foto: SAPPO-UAB).

fosa o pequeña canalización se caracteriza en términos generales, por tener una sección semicircular o ligeramente convexa, de profundidad variable pero nunca superior a los $20 \mathrm{~cm}$. Las paredes y la base de las misma presentan elementos constructivos sobre todo al disponer en varios casos, un revestimiento de losas calcáreas dispuestas de manera vertical que delimitan una pequeña depresión por dónde discurre el agua. La base tiene una morfología convexa o apuntada en algunos casos mientras que en otros es plana y revestida por losas en algún caso o por restos orgánicos que indicarían la existencia de algún tipo de protección de esta base. En algunos casos la documentación de estructuras de silicatos indica la presencia de madera (fig. 3).

El recorrido de estos pequeños canales es muy interesante pues se ha podido reseguir en varios casos y, en la mayoría de las evidencias, tienen una longitud de varios metros (aproximadamente de 1,5 metros a 4 metros, en los ejemplos mejor conservados), siempre ubicadas en el callejón o espacio abierto que separa varias casas con una disposición en paralelo al muro exterior. Muy interesante es la dirección de los mismos, pues en los documentados en las fases FO 11,12 estarían orientados hacia el norte del tell, adentrándose en el corte de la excavación. La campaña de excavación de 2011 permitió documentar un caso al contrario (FO 10) en el cual la dirección era hacia el sur del tell, permitiendo conocer el tramo final y el sistema de finalización del "recorrido" de los citados canales.

En efecto en la última campaña, de excavación, en 2011, se pudo excavar en amplitud el espacio abierto asociado a una de estas zonas de deposición de uno de estos 
canales y en él se localizó el final de la fosa y sobretodo una concentración de sedimentos de coloración grisácea en un espacio de 2,30 × 2,10 metros dónde se identifica la perduración del nivel de concentración de sedimento orgánico (4ICla). Concretamente es el proceso de colmatación de los residuos procedentes de la evacuación de aguas de una de las casas de esta fase (casa 4IC). Esta excavación en extensión ha permitido recuperar, a partir de los datos de las campañas precedentes, otras estructuras de este tipo que no se habían podido interpretar y que con la documentación actual permiten a una revisión más válida de los usos de los espacios abiertos en el poblado y a los procesos de erosión y colmatación vinculados a los niveles de circulación y acceso a las casas del poblado. Es verdad que una finalización tan poco delimitada de estos "canales" de desagüe domestico sorprende, sobre todo después de la inversión de trabajo observada en la propia conducción y en el resto de arquitectura doméstica.

Este tipo de estructuras vinculadas con la evacuación de aguas, dentro del espacio doméstico, está muy poco documentado en los poblados neolíticos del Próximo Oriente y estos hallazgos son realmente excepcionales. Por si singularidad hay que considerarlos indicadores de la sofisticación arquitectónica de las casas y del uso del espacio en los poblados neolíticos que se encuentran en la fase de consolidación de las nuevas formas de producción.

Probablemente uno de los paralelos que indican una estructuración similar a los canales o conductos complejos asociados a las casas sea el asentamiento de El Kowm-2 /Caracol (Cubeta d'El Kowm, Palmira, Siria) (Stordeur, 2000). Este interesante yacimiento de cronología ligeramente más reciente que Halula, pues está datado en el primer cuarto del VII milenio ANE, proporciona unas evidencias de conducción de agua en el propio interior de las casas en un contexto de renovación de modelos y técnicas arquitectónicas. Recordemos que se trata de un asentamiento de tipo pre-cerámico, en la zona semiárida de la Siria interior.

Es interesante ver, en primer lugar, las estructuras exteriores, ya que encontramos como en el caso de Halula, un reguero o canalización exterior ("rigole") construida en la mitad de una callejuela o espacio de circulación entre dos casas (casas IB y IX). Su sección es semicircular, con una anchura de unos $10 \mathrm{~cm}$, constituyendo una verdadera construcción que desemboca sobre el espacio exterior que rodea las habitaciones. Un adobe puesto de manera transversal sobre la parte final de la canalización indica su terminación. Los autores del estudio detallado indican que se ignora cómo se comporta esta evacuación de agua cuando la callejuela se rellena progresivamente por la acumulación del detritus. En efecto, en el análisis del espacio exterior de las construcciones se documenta una sucesión estratigráfica en la cual se alternan los depósitos de detritus y aportaciones antrópicas de enlucidos de arcillas o pequeños empedrados realizados con fragmentos de vajilla blanca conocida en la literatura como Vaiselles Blanches, destinados al drenaje o saneamiento y que realzan de manera significativa (más de $50 \mathrm{~cm}$.) el nivel de los suelos exteriores. Esto evita que se puedan producir problemas de erosión por el agua en la parte inferior del muro, parte siempre más delicada de cara a los procesos de erosión, humedad e inestabilidad. El canal está relleno por una mezcla de detritus ceniciento donde se han recuperado muchos restos de fauna, algunos de los cuales están enteros, no constatando la misma alternancia que se citaba en el exterior (estratos de detritus con estratos de arcilla o fragmentos de Vaiselles Blanches).

Las estructuras de gestión del agua en el interior de las casas se describen como elementos construidos sofisticados, estando localizados en dos construcciones denominadas "casas con pequeñas células comunicantes" (Stordeur et al., 2000). Se distinguen tres tipos de estructuras.

En primer lugar, los regueros o pequeños canales laterales en el umbral de la habitación. Los arqueólogos consideraron que no sería un agujero, sino un espacio de evacuación situado en el propio umbral.

El segundo tipo es el agujero de evacuación abierto en un muro. Este tipo no aparece solo, sino que se combina con otros para formar un conjunto que podría funcionar de forma sincrónica. En un caso, la casa IX-IV, el agujero está realizado en la base de un muro que es la culminación de una canal excavada en el suelo. El conjunto está enlucido de yeso, que permite circular el agua de una habitación a otra, dada su situación inferior o en contrabajo. Otro orificio, situado a algunos centímetros más altos en el mismo muro, permitiría mejorar la circulación de aire en esta habitación muy exigua y en la cual se documenta un gran hogar. Finalmente otro agujero de evacuación, permite al agua salir al exterior de la casa.

En una de las casas ("Maison XII") se detecta un sistema combinado de circulación y de evacuación de agua. Se inicia por un agujero de apertura realizado en la base del umbral en el interior, continuando debajo el enlucido del suelo en forma de conducto subterráneo, para reaparecer y transformarse en reguero cruzando por fin un muro para desembocar en el exterior. El trayecto es oblicuo y paralelo a la pared externa del muro, siendo el recorrido total de unos 150 centímetros (Stordeur et al., 2000: 85).

Se trata como se ha podido comprobar de unos sistemas realmente complejos, bien estructurados y con unas construcciones con alta inversión técnica, algunas de ellas con cierto refinamiento como el hecho que el sistema sea mitad subterráneo mitad en reguero o canal y aparece como una variable importante en la concepción de la evacuación del agua. Estas evidencias demuestran la inversión concedida a los sistemas de gestión de aguas, sin llegar a definirse su uso como agua potable o residual, en las propias construcciones de hábitat de los poblados neolíticos.

\subsection{Sistemas de canalizaciones en espacios abiertos en los poblados}

Las evidencias de construcciones para la circulación de agua, sin estar directamente asociado a construcciones domesticas se ha documentado también en Tell Halula, 


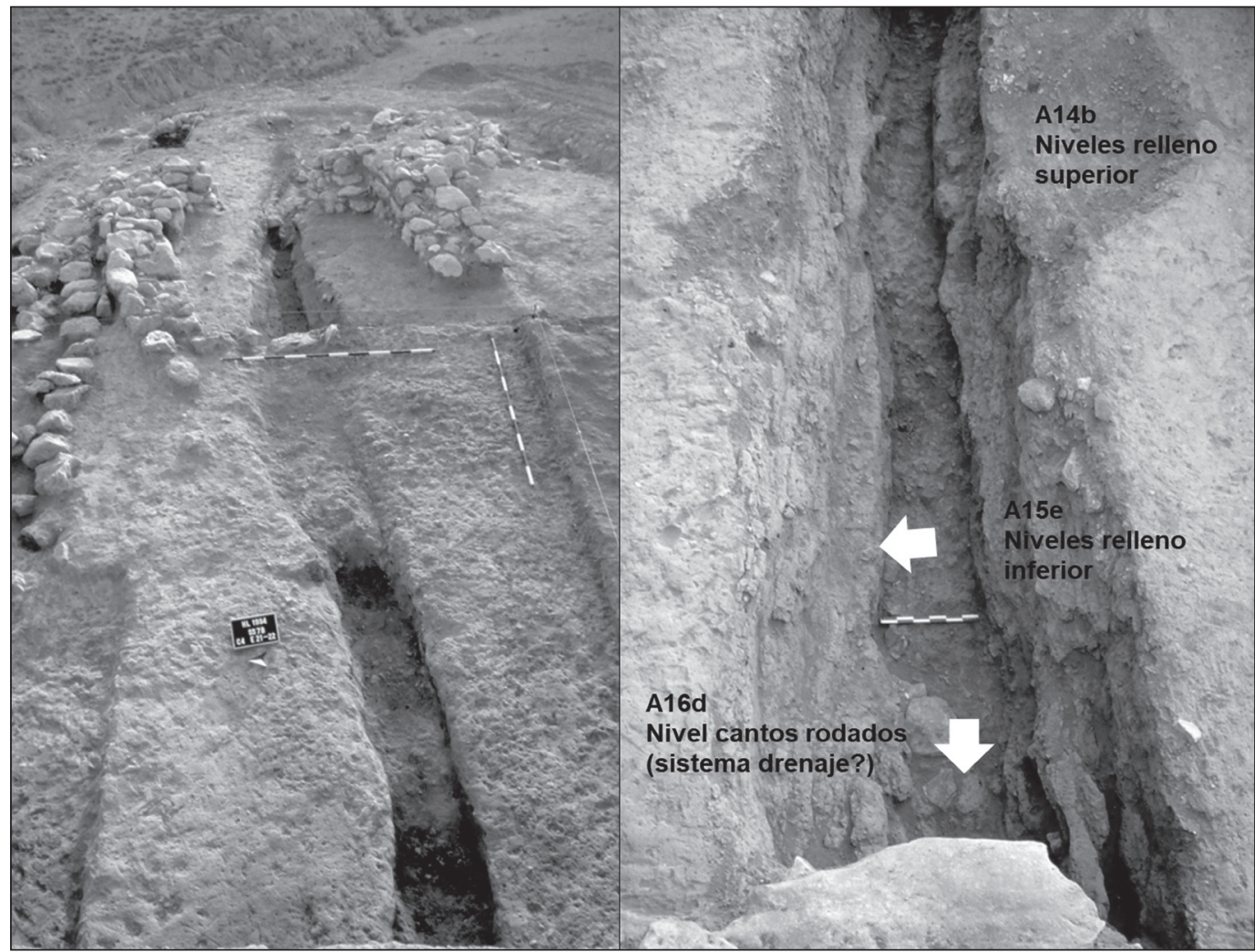

Figura 4. Tell Halula.- Sector S7, Canal, Pre-Halaf (c. 6.700 cal BC), Visión general del canal excavado. (Foto: SAPPO, UAB) (Foto: SAPPO-UAB).

concretamente en el sector SS 7 situado en la zona este del yacimiento con una cronología se sitúa en el periodo Pre-Halaf (c. 6700 cal BC) ${ }^{5}$.

Esta área del yacimiento constituye un gran espacio abierto situado al límite nordeste del poblado. La excavación puso en evidencia, en uno de los niveles SS7-VI, un conjunto de estructuras definidas por una parte por amplias superficies de ocupación (suelos de tierra batida) con abundantes reutilizaciones y un mínimo de tres reutilizaciones por suelo preparado. Por otra parte tres grandes muros de piedra (Muros E10 y E6 y E4) definen una forma rectangular de espacio exterior, donde se ubican los suelos citados anteriormente, y que interpretamos como el muro de cierre o muralla del asentamiento. No hay restos de construcciones domésticas sino amplios espacios abiertos, con unos suelos en tierra batida, bien construidos con una base de pequeños guijarros (Molist, 1996; Molist, 1998).

Es en esta zona dónde se ha documentado una estructura construida, que se interpreta como una canalización, constituyendo una de las evidencias más antiguas de este tipo de estructuras. Se trata de una fosa excavada en distintos suelos de circulación que presenta una forma alar-

5 En esta zona se excavó, entre los años 1992 y 1994, en una superficie de $228 \mathrm{~m} 2$. Los trabajos pusieron en evidencia una sucesión de trece niveles caracterizados por las preparaciones, construcciones y abandonos de grandes conjuntos arquitectónicos (Molist 1996; Molist y Vicente, 2013; Molist et al., 2014). gada/rectangular, de poca anchura pero que en longitud se ha podido reseguir en un total de 22 metros (fig. 4). Esta fosa presenta un tratamiento cuidado en paredes y del fondo (revestido de arcilla, con un pequeño zocolo de guijarros a la base) y su cubierta con losas calcáreas planas, que indican por una parte el acabado cuidado de la construcción y la importancia del mismo dado que se han constatado tres reparaciones de la misma. Esta estructura se ha interpretado como una canalización con drenaje, probablemente para la evacuación de las aguas usadas dado que la suave pendiente de la estructura tiene una dirección oeste hacia este, es decir del interior hacia el exterior (fig. 5 y 6).

Los niveles arqueológicos superiores de esta misma área, muestran una gran continuidad en el uso de este espacio y en algunos de ellos la presencia de la canalización es una constante. Así, los niveles SS7-X y SS7-XI, ofrecen, de nuevo, la constatación de la construcción y uso de una estructura arquitectónica formada por un gran muro de dirección Este-Oeste, uno en su fase original (nivel SS7-X) y el otro en su reconstrucción / reparación (SS7-XI).

De nuevo se trata de suelos en tierra batida, que ocupan un espacio asociado a un muro (E10) con mayores evidencias del uso antrópico de estos grandes espacios abiertos, pues se han documentado dos silos y una probable construcción complementaria, formada por un muro perpendicular al E10, no excavado en su totalidad. En el nivel superior se documentó una fosa alargada de carac- 


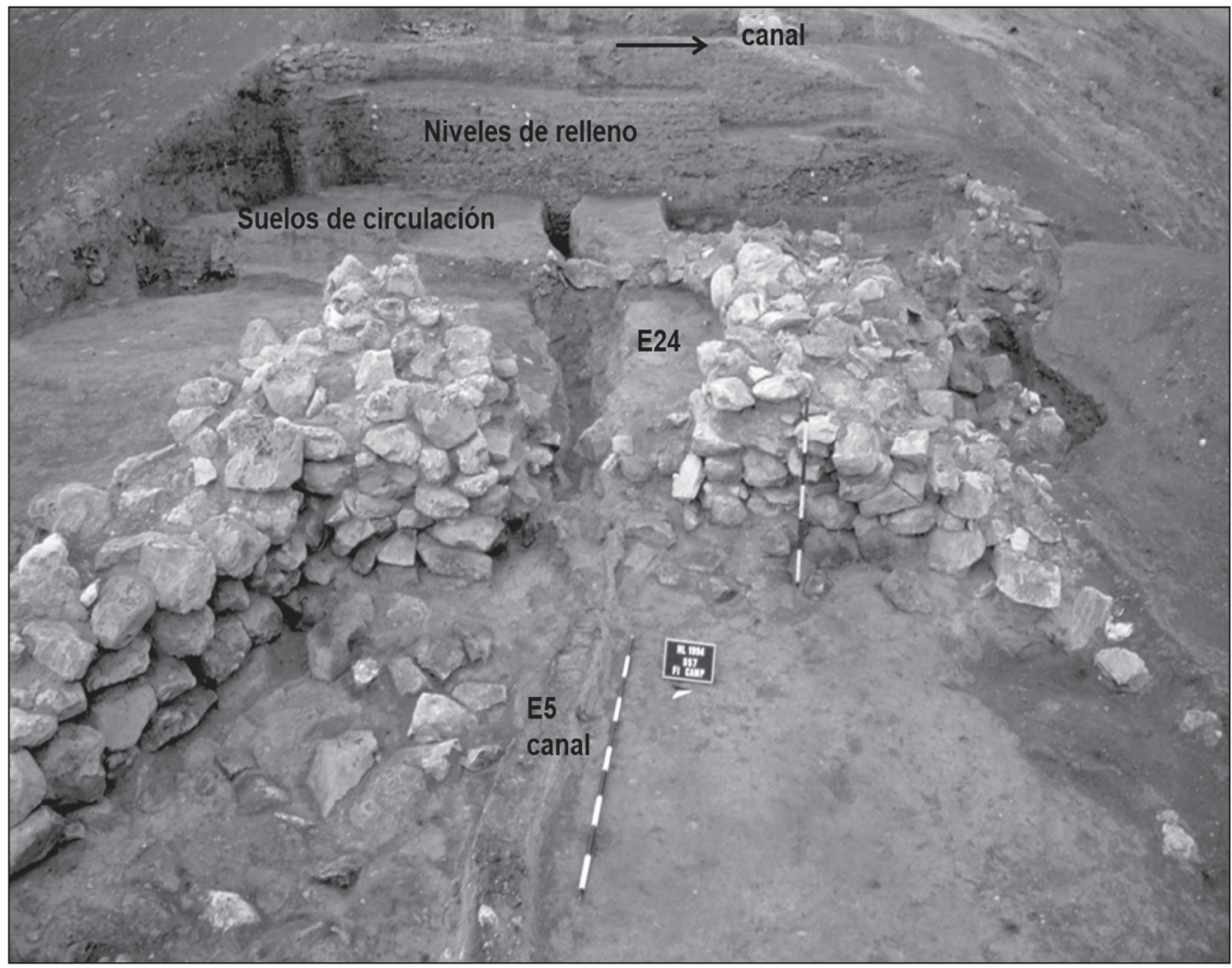

Figura 5. Sector SS7, Canal Pre-Halaf (c. 6.700 cal BC). Visión general desde del este, con los muros de cierre del poblado, el espacio interior y el canal excavado en los suelos de este espacio. (Foto: SAPPO, UAB).

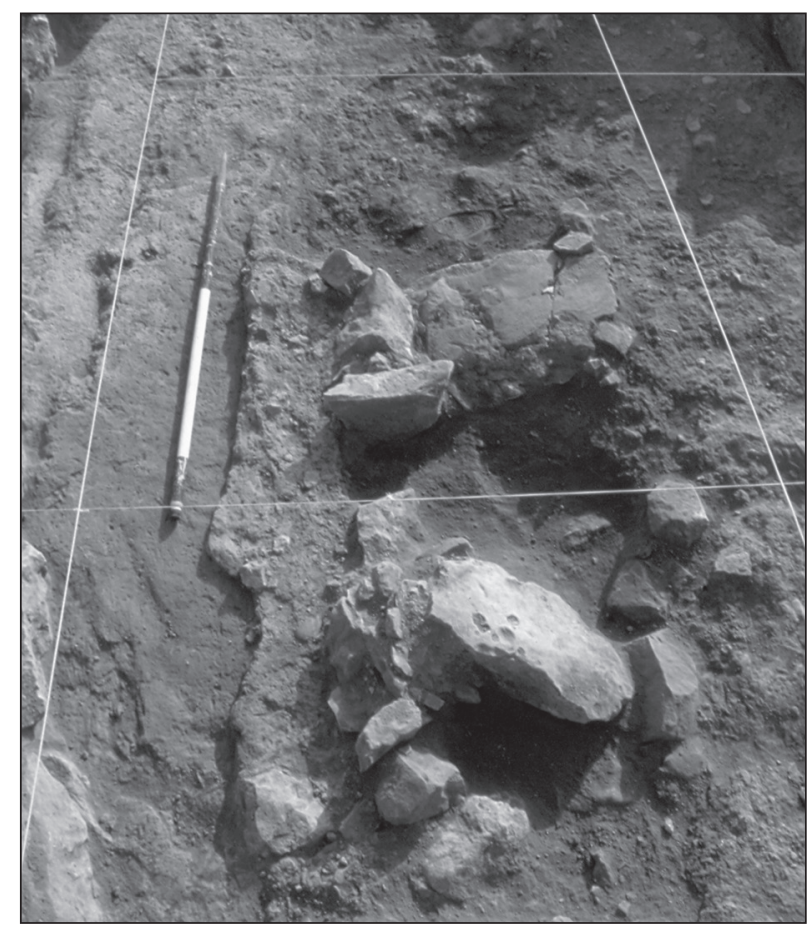

Figura 6. Sector SS7, Canal Pre-Halaf (c. 6.700 cal BC). Detalle de las losas planas que cubren el canal. (Foto:SAPPO, UAB).

terísticas similares a las del nivel SS7-VI, a pesar que la inversión de trabajo es menor, fue interpretada igualmente como una canalización (fig. 5).
Hay que destacar que este último testimonio es singularmente importante dado que constituye de nuevo una evidencia de construcciones construidas a gran escala destinadas circulación del agua.

\section{Consideraciones finales}

La complejidad de los poblados neolíticos del Próximo Oriente está aún por completar tanto a nivel de técnicas constructivas como sobretodo de usos y caracterización social de los espacios. La gestión de los recursos hídricos, por su importancia no sólo para todo grupo humano, sino también para los animales domesticados y los cultivos es fundamental para el mantenimiento del grupo. En este sentido la diversidad de acceso y gestión a este recurso muestra la gran capacidad de resiliencia de estas comunidades. Las evidencias documentadas muestran una capacidad organizativa que supera la esfera privada y que a nivel tecnológico, simbólico y social requiere de una pericia permanente (captación, potabilidad, distribución, evacuación...) del conjunto de la comunidad.

En la literatura esta complejidad queda plasmada en la documentación de la gestión del agua sin acotar, definir e identificar la inversión de tiempo, recursos y materiales que estas variables pueden presentar, así como la respuesta individualizada de poblado en poblado. A partir del estudio del asentamiento de tell Halula se ha podido inferir en algunos aspectos que, a nuestro entender son realmente 
importantes para entender y caracterizar las estrategias adoptadas por los primeros agricultores y ganaderos.

Por una parte, es importante remarcar la antigüedad de los sistemas constructivos destinados a la gestión del agua en el entorno doméstico, prácticamente des de los inicios de los primeros poblados agrícolas.

Por otra parte, la constatación de su desarrollo paralelizable con el de las técnicas constructivas y la complejidad de los espacios de habitación y modelos constructivos. Aspecto relacionable con la propia constitución de la comunidad agropecuaria. Su existencia tiene un espacio en el salto cualitativo que se documenta por ejemplo en los poblados agrícolas del VIII milenio (MPNNB).

Es quizá también sorprendente que una parte de estas estructuras estén aparentemente destinadas a la evacuación del agua usada más que en la captación de la misma. Aspectos como la gestión de los residuos y los problemas sanitarios podrían ser una explicación plausible para la aparición y desarrollo de estas estructuras. Evitar enfermedades contagiosas en relación a la convivencia entre animales domésticos y grupo humano en los mismos espacios (Baker et al., 2017; Anfruns et al., 2013). En la misma línea interpretativa, se ha propuesto la aparición y consolidación de los enlucidos en cal o yeso en las construcciones domésticas del periodo neolítico pre cerámico.

A nivel tecnológico, para estas mismas construcciones, se considera que se trata de evidencias de estructuras pensadas y construidas de manera específica para este tipo de instalaciones, con un uso claramente doméstico. Por otra parte y en el caso de las casas de Tell Halula (MPPNB) esto se evidencia por la regularización o normativización y el recurso sistemático de este tipo de instalaciones en cada una de las casas del poblado. Es también muy interesante para la configuración de los usos de los espacios, interiores y exteriores y la constatación de la acumulación de las aguas usadas en los espacios abiertos exteriores. Esta variable permitirá inferir en la accesibilidad y direccionalidad de los niveles de circulación entre las casas y en el resto del poblado.

Finalmente destacar las evidencias de canalizaciones colectivas construidas de manera prolongada, en amplios espacios exteriores durante la primera mitad del VII milenio. Estas constituyen una evidencia suplementaria de la necesidad de estructuración y gestión de los espacios abiertos o áreas colectivas del poblado. Mostrando, una vez más, la capacidad tecnológica precedente al gran salto tecnológico que representará la utilización de la irrigación para la producción agrícola que se desarrollará en los siguientes milenios.

La documentación presentada y las hipótesis de su utilización, sobretodo en relación a su uso para la evacuación de aguas usadas, necesita ser contrastada de manera más eficiente. Así se han retomado recientemente los análisis micromorfológicos de las muestras procedentes de las acumulaciones sedimentológicas de las zonas de vertido de las aguas usadas en el poblado del M/PPNB ${ }^{6}$. Una

${ }^{6}$ En curso de realización por Julia Wattez INRAP, Paris (Francia). continuidad en la investigación con la aplicación de técnicas arqueométricas y de nuevas tecnologías, que resulta imprescindible en el momento actual de la arqueología para generar nuevo conocimiento.

\section{Bibliografía}

Anfruns, J.; Estebaranz-Sánchez F.; Martinez Martinez L.M. y Pérez-Pérez A. (2013): "La población neolítica de Tell Halula. Estudio antropologico (campañas 19952005)". En M. Molist (ed.): Tell Halula: un poblado de los primeros agricultores en el valle del Éufrates, Siria. Ministerio de Educación y Ciencia, Dirección General de Bienes Culturales, Instituto Patrimonio Histórico Español. Vol 2: 440-451.

Araus J.L.; Febrero A.; Catala M.; Molist M.; Voltas J. y Romagosas I. (1999): “Crop Water availability in early agriculture: evidence from carbon isotope discrimination of seeds from a tenth millennium BP site on the Euphrates". Global Change Biology, 5: 201-212. DOI: https://doi.org/10.1046/j.1365-2486.1999.00213.x

Aurenche, O. (1981): La maison orientale. L'architecture du Proche-Orient ancien des origines au milieu du quatrième millénaire. Librairie Orientaliste Paul Genthner.

Aurenche, O. (1982): "Préhistoire des sociétés hydrauliques du Proche-Orient ancien. L'homme et l'eau en méditerrannée et au Proche Orient II Aménagements hydrauliques, état et legislations". En J.Y. Métral y P. Sanlaville (eds.): L'homme et l'eau en Méditerranée et au Proche-Orient. Travaux de la Maison de l'Orient, 3: 7-30.

Baker, O.; Chamel, B.; Coqueugniot, E.; Khawan, R.; Stordeur, D.; Perrin, P.; Falfi, G.; Gourichon, L.; Coqueugniot H.; Le Mort, F. y Dutour, O. (2017): "Prehistory of human tuberculosis: Earliest evidence from the onset of animal husbandry in the Near East". Paleorient, 43 (2): 35-51.

Braidwood, R.J. y Braidwood, L.S. (1960): Excavations in the Plain of Antioch. The Earliest assemblages Phases $A$-J. Oriental Institute Publications, vol. 32, The University of Chicago.

Buxó R. y Rovira N. (2013): “Tell Halula: arqueobotánica de los restos de semillas y frutos". En M. Molist (coord.): Tell Halula: un poblado de los primeros agricultores en el valle del Éufrates, Siria. Memoria Científica - Tomo I y II. Ministerio de Educación, Cultura y Deporte. Secretaria General Técnica. Subdirección General de Documentación y Publicaciones. Madrid: 359-389.

Cauvin, J. (1981): “Le "problème de l'eau" au Proche-Orient, de l'homme prédateur aux premières sociétés hydrauliques". En J. Métral y P. Sanlaville (eds.): L'homme et l'eau en Méditerranée et au Proche-Orient. Travaux de la Maison de l'Orient, 2: 20-30.

Cauvin, J. (1994): Naissance des divinités, naissance de l'agriculture: La révolution des symboles au Néolithique. CNRS Éditions, Paris. 
Cruells W. (2013): “La cerámica Halaf en Tell Halula VII y Vi milenios cal BC. Orígenes y desarrollo". En M. Molist (ed.), Tell Halula: un poblado de los primeros agricultores en el valle del Éufrates, Siria. Ministerio de Educación y Ciencia, Tomo II: 212-231.

De Contenson, H. y Van Liere, W. (1966): "Seconde campagne à Tell Ramad. Rapport Préliminaire”. Annales Archéologiques Arabes, Syriennes, 16: 167-174.

Dunand, M. (1973): Fouilles de Byblos. L'architecture, les tombes, le matériel doméstique, des origines néolithiques à l'avènement urbain. Paris.

Faura J.M. (2016): El conjunt ceràmic del VII mil·lenni cal bc de Tell Halula (Síria). Una evidència dels processos de canvi socioeconòmic. Phd, Departament de Prehistòria. Universitat Autòma de Barcelona. http:// www.tesisenred.net/handle/10803/399329.

Ferrio, J.P.; Arab, G.; Buxó R.; Guerrrero, E.; Molist M.; Voltas, J. y Araus J.L. (2011): “Agricultural expansion and settlement economy in tell Halula (Mid Eufrates Valley) A diachronic study from early Neolithic to present". Journal of Arid Environments, 86: 104-112. DOI: https://doi.org/10.1016/j.jaridenv.2011.09.011

Fujii, S. (2007): "Wadi Abu Tulayha and Wadi Ruweishid ash-Sharqi: An Investigation of PPNB Barrage Systems in the Jafr Basin". Neo-Lithics, 2 (7): 6-17.

Fujii, S. (2010): "Domestication of Runoff Surface Water: Current Evidence and New Perspectives from the Jafr Pastoral Neolithic". Neo-Lihtics, 2 (10): 17-32.

Galili, E., Weinstein-Evron, M., Hershkovitz, I., Gopher, A., Kislev, M., Lernau, O., Horrowitz, L. K y Lernau, H. (1993): "Atlil-Yam: A Prehistoric Site on the Sea Floor of the Israel Coast". Journal of Field Archaeology, 20: 133-157.

Garfinkel, Y.; Vered A. y O. Bar-Yosef, O. (2005): “The Domestication of Water: The Neolithic Well of Sha' ar Hagolan, Jordan Valley, Israel". Antiquity, 80: 686-696. DOI: https://doi.org/10.1017/S0003598X00094138

Gómez-Bach A. (2011): Caracterización del producto cerámico en las comunidades neolíticas de mediados del VI milenio Cal BC: El valle del Éufrates y el valle del Khabur en el Halaffinal (Siria). Thèse de doctorat. Université de Liège.

Guilaine, J. (2003): "Parekklisha-Shillourokambos périodisation et aménagements domestiques". En J. Guilaine y A. Le Brun (eds.): Le Néolithique de Chypre. École Française d'Athènes, $\mathrm{BCH}$, supplément 43: 3-14.

Guilaine, J.; Briois, F. y Vigne, J-D. (2011): Shillourokambos. Un établessiment néolithique pré-cerámique à Chypre. Les fouilles du secteur 1. Éditions Errance et École française d'Athènes.

Lloyd, S.; Safar, F. y Braidwood, R. (1945): “Tell Hassuna Excavations by the Iraq Government Directorate General of Antiquities in 1945-1944". Journal of Near Eastern Studies, 4 (4): 255-289. DOI: https://doi. org/10.1086/370765

Mellaart, J. (1965): Earliest Civilizations of the Near East. Thames and Hudson.

Mellaart, J. (1970): Excavations at Hacilar. Occasional publications of the British Institut of Archaeology at Ankara. Edinburg University Press.
Molist, M. (1998): "Espace collectif et domestique dans le néolithique des IX éme et VIII éme millénaires B.P. au nord de la Syrie: apports du site de Tell Halula (Vallée de l'Euphrate)". En O. Aurenche y M. Fortin (eds.): Espace Naturel, Espace Habité en Syrie du Nord. (10-2 1/4 millenaires av. J. C.). Université de Labal-Quebec y Gremo (Lyon, Francia). Quebec, (Canada): 115-130.

Molist, M. (ed.) (1996): Tell Halula (Siria). Un yacimiento Neolítico del Valle Medio del Éufrates. Campañas de 1991 y 1992. Ministerio de Educación y Cultura. Madrid.

Molist, M. (ed.) (2013): Tell Halula: un poblado de los primeros agricultores en el valle del Éufrates, Siria. Ministerio de Educación y Ciencia, Dirección General de Bienes Culturales, Instituto Patrimonio Histórico Español.

Molist, M. y Vicente, O. (2013): “Tell Halula 1995 - 2005. Análisis estratigráfico y arquitectónico de los diferentes horizontes históricos de Tell Halula”. En M. Molist (coord.): Tell Halula: un poblado de los primeros agricultores en el valle del Éufrates, Siria. Memoria Científica - Tomo I y II. Ministerio de Educación, Cultura y Deporte. Secretaria General Técnica. Subdirección General de Documentación y Publicaciones, Madrid: 31-86.

Molist, M.; Gómez, A.; Bofill, M.; Cruells, W.; Faura, J.M.; Marchiori, C. y Martín, J. (2014): "Maisons et constructions d'habitation dans le Néolithique. Une approche de l'évolution des unités d'habitat domestiques à partir des documents de Tell Halula (Vallée de l'Éuphrate, Syrie)". En J.L. Montero (ed.): Redonner vie aux Mésopotamiens. Mélanges offerts à Jean-Claude Margueron à l'occasion de son 80è anniversaire. Publicaciones de la Universidad de la Coruña: 97-116.

Peltenburg E. (2003): "Identifying Settlement of the X-IX Millenium B.P. in Cyprus from the contens of Kissonerga-Mylouthkia Wells". En J. Guilaine, A. Le Brun (eds.): Le Néolithique de Chypre. École Française d'Athènes, $\mathrm{BCH}$, supplément 43. Athènes: 15-33.

Stordeur, D. (ed.) (2000): El Kowm 2, Une île dans le désert. La fin du Néolithique précéramique dans la steppe syrienne. CNRS, editions.

Stordeur, D.; Marechal, L. y Molist, M. (2000): "Les données architectoniques". En D. Stordeur (ed.): El Kowm 2, Une île dans le désert. La fin du Néolithique précéramique dans la steppe syrienne. CNRS, editions: 35-87.

Tornero, C. y Saña, M. (2010): "Integrating stable isotopes to study the origin of management strategies of domestic animals, $\delta 13 \mathrm{C}$ and $\delta 18 \mathrm{O}$ results from bioapatite enamel of cattle from Tell Halula site, Syria (7800-7000 cal BC)". En I. Turbanti-Memmi (ed.): Proceedings of the 37th International Symposium on Archaeometry, 13th-16th May 2008. Siena, Italy, Berlin. Springer: 435-440.

Willcox, G.; Buxó, R. y Herveux L. (2009): "Late Pleistocene and Early Holocene climate and the beginnings of cultivation in northern Syria". The Holocene, 19 (1): 151-158. DOI: https://doi. org/10.1177/0959683608098961 